\title{
Glial degeneration as a model of depression
}

\author{
Maria Śmiałowska\#, Bernadeta Szewczyk, Monika Woźniak, \\ Anika Wawrzak-Wleciał, Helena Domin\# \\ Department of Neurobiology, Institute of Pharmacology, Polish Academy of Sciences, Smętna 12, \\ PL 31-343 Kraków, Poland \\ Correspondence: Maria Śmiałowska, e-mail: nfsmialo@cyf-kr.edu.pl; Helena Domin, \\ e-mail: domin@if-pan.krakow.pl
}

\begin{abstract}
:
Major depression (MD) is a common and disabling disorder but knowledge of its pathophysiology is still incomplete. In the last years, degenerations or dysfunctions of glial cells, especially astrocytes, have been postulated to play a critical role in the pathogenesis of depression. Glial loss in prefrontal and limbic brain regions was observed in depressed patients and in animal models of stress and depression. Degeneration of astrocytes resulted in an excess glutamate in the synaptic cleft and glutamate/GABA imbalance in the affected structures. This review presents an up-to-date information concerning the role of glial cells in maintenance of glutamate/GABA balance in the brain tripartite glutamatergic synapses; discusses the importance of glial pathology and presents models of depression based on astrocyte impairment. The model of degeneration of astrocytes in the medial prefrontal cortex of the rat, induced by the specific astrocytic toxin $\alpha$-aminoadipic acid, is presented as a valuable model for studying antidepressant compounds.
\end{abstract}

Key words:

depression, astrocyte degeneration, gliotoxin, glutamate/GABA balance

\section{Introduction}

Major depression is one of the most prevalent forms of mental illnesses and is among the leading causes of disability, affecting about 121 million people worldwide. Approximately $30 \%$ of patients fail to respond to the present therapies. Low efficacy of treatment is partially caused by the heterogeneous nature of this disorder, that is influenced by multiple molecular, environmental and genetic factors, some of which are not fully understood. Several hypotheses including chronic stress, failure of adulthood neurogenesis, altered neuroplasticity, dysfunction of monoaminergic systems and genetic factors have been studied to elucidate depressive-related behaviors [31, 35]. Monoamine hypothesis of depression has dominated our understanding of both the pathophysiology of depression and the action of pharmacological treatments for the last decades and it led to production of several generations of antidepressant agents [32]. In the last several years, the focus of research has shifted from monoamines toward other molecular mechanisms, including glutamatergic neurotransmission [10].

In the mammalian brain, glutamate is the most abundant and major excitatory neurotransmitter, and glutamic acid-induced excitation is in a balance with the inhibitory action of $\gamma$-aminobutyric acid (GABA),

\footnotetext{
\# contributed equally to this article
} 


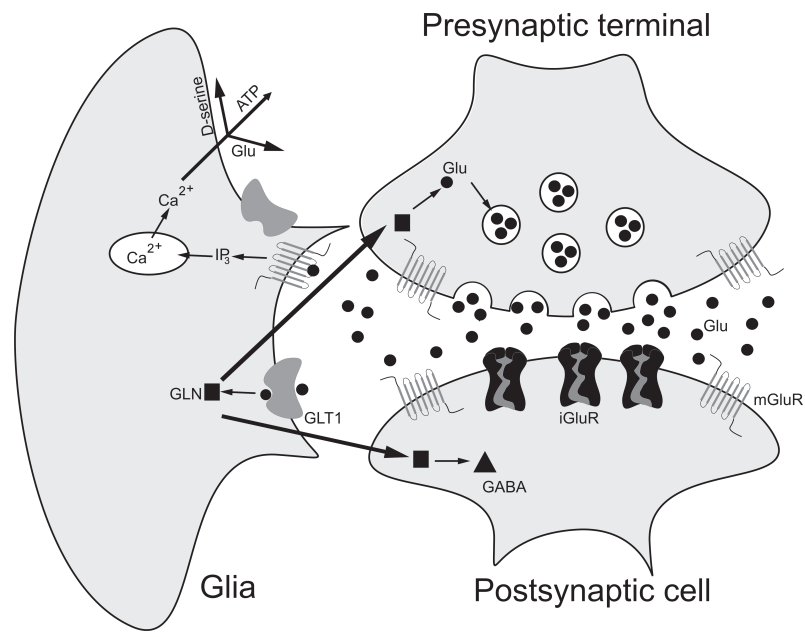

Fig. 1. A schematic drawing of glutamatergic tripartite synapse formed by presynaptic glutamatergic terminal, postsynaptic GABA neuron and astrocyte processes (Glia) surrounding the synapse. Only some, chosen elements of bidirectional communication between glia and neuronal elements were shown. In the glutamatergic synapse astrocytes remove the excess glutamate (Glu) from the synaptic cleft through the specific membrane transporter GLT1, modulating Glu concentration in the cleft. Glutamate released from the glu tamatergic terminals is taken up by glial cell, where it is metabolized to glutamine (GLN) and released. Then, GLN may be taken up by Glu or GABA terminals where it is converted to an appropriate neurotransmitter. Besides being taken up, Glu released by nerve terminals activates receptors on the glial cell membrane which induces mobilization of calcium ions and release of gliotransmitters: Glu, ATP, D-serine, and others

which is the main inhibitory amino acid neurotransmitter in the brain. An imbalance between excitation and inhibition has been observed in the brains of patients with mental diseases, such as anxiety, depression and schizophrenia. Depression and anxiety have been postulated to be connected with an increasing glutamatergic excitation and decreasing GABAergic inhibition. The increased level of glutamate was observed in the brains and CSF of patients diagnosed with depression [20]. Glutamatergic neurotransmission is strongly involved in the pathophysiology of depression and in the therapeutic action of the majority of antidepressant drugs [17]. It is postulated that reduced glutamatergic transmission may be an underlying mechanism of antidepressant effects of such drug as phenelzine [57], an antidepressant/antipanic drug which attenuates glutamatergic transmission. Thus it has been suggested that antidepressants may exert their effects by attenuating the release of glutamate and/or postsynaptic excitability [41]. The pharmacological support for the glutamatergic theory of depression comes also from studies employing
NMDA receptor antagonists. It was described for the first time by Trullas and Skolnick [53] that functional antagonists of NMDA receptor exhibit antidepressantlike activity. Adaptive changes in NMDA receptors have been suggested to be the final common pathway for antidepressant action [51]. The NMDA receptor antagonist ketamine or the NMDA channel blocker AZD6765 given in a single dose, can reverse depressive symptoms in treatment-resistant depressive patients [9].

As glial cells, especially astrocytes, play a very important role in maintenance of the glutamate/GABA balance, we focused our attention on the newly introduced experimental model of depression induced by astrocyte depletion. We verified if such model really corresponds to behavioral and cellular changes in depression and if it may be useful for studying antidepressant drugs.

\section{The role of glial cells in glutamate/GABA balance in the brain}

It is generally assumed that glia are very numerous cells in the mammalian brain and the neurons/glia ratio fluctuates depending on the species and brain structure (in the cerebral cortex between 0.3 to 4.4 depending on cortical layer) [43]. Glial cells, first described in 1860 by Virchow and named neuroglia, were for a long time thought to be only supporting elements for neurons. However, over the years, researchers have found that glial cells interact with neurons much more actively. Glial cells in the central nervous system (CNS) can be divided into three main types: astrocytes, oligodendrocytes (both of ectodermal origin and called macroglia) and microglia (of mesodermal origin belonging to the monocytemacrophage lineage). Microglia perform immunomodulatory functions in response to injury or disease. Oligodendrocytes ensheath long axons with myelin mainly in white matter or play a role in trophic and nutritional support as a perineuronal oligodendrocytes in gray matter. Astrocytes, the most numerous type of glial cells, transfer nutrients and other substances between the neurons and the bloodstream, play a central role in building and maintaining the blood-brain barrier, guide the migration and differentiation of neurons, take part in the regulation of energy metabolism, regional blood flow, neurotrophin production and, 
moreover, they take up many transmitters from the extracellular fluid and possess receptors for them [43]. The regulation of the local concentration of ions and neuroactive substances is a very important function of astrocytes [25] which is crucial for keeping CNS homeostasis. Bidirectional communication between neurons and astrocytes was revealed by many research groups during 1990s and term ,tripartite synapse" was proposed because the astrocytes are the third element of the synapse [2, 45] (see Fig. 1).

Astrocytes are an integral part of tripartite synapse where they regulate neuronal excitability. In the glutamatergic synapses, the most abundant type of synapses in the CNS, astrocytes control the levels of extracellular glutamate, the main excitatory neurotransmitter in the mammalian brain [56], so they maintain the balance between the excitatory and inhibitory neurotransmission therein.

\section{Glial cell pathology in depression}

Postmortem studies of brains of depressed patients showed a decrease in the density of glial cells in cortical regions, especially in the prefrontal and cingular areas, which was one of the most consistent findings [47]. Among glial cells, astrocyte pathology is a prominent feature of mood disorders. Reduced levels of glial fibrillary acidic protein (GFAP), a marker of astrocytes in the CNS, was found in some cortical and limbic structures of depressed subjects [33, 50] and in animal models of depression [18]. The increase in the level of S100B protein, a marker of glial degenerations in blood serum of patients correlated with exacerbation of depressive symptoms and was inhibited by antidepressants [49]. Also gene expression studies of human postmortem tissue provided additional evidence for the astrocytic pathology in depression [47]. Most of evidence indicated that in patients suffering from mood disorders, the strongest glial cell pathology was seen in the prefrontal cortex $[34,42,54]$. The decrease in the density of astrocytes or reduced GFAP level was also found in the amygdala and cerebellum of patients suffering for major depression in comparison to bipolar disorder, schizophrenia or control brains [1, 7, 16]. However, there are also studies which do not confirm glial degeneration in affective disorders [14], but those results concern the other cortical structure, entorhinal cortex. It has been postu- lated that the discrepancy in the results in such glial changes may depend on patients' age. MiguelHidalgo and coworkers [33] demonstrated that the decrease in GFAP-ir level was found in younger (30-45 years old) but not in older (46-86 years old) patients. The lack of a significant decrease in astrocytic density when we compare old MDD patients with old control subjects may arise from the fact that in old patients astroglial marker GFAP has much higher level than in young subjects as a result of aging-related appearance of many reactive astrocytes. Similarly, no changes in glial density was found in the orbitofrontal cortex and anterior cingulate cortex in elderly MDD patients. Therefore, aside from some discrepant results connected with aging, inflammation and other neuropathological states, it is generally accepted that the reduction in astrocytes and related markers (GFAP, S100B, connexin 40, 43, aquaporin, glutamate transporters) is a key features in the pathology of MDD (for review see [44]). Besides of astrocytes, a few studies evidenced a significant oligodendrocytic pathology in mood disorders $[15,19,54,55]$.

As mentioned above, the uptake of released glutamate is an important function of astrocytes in the brain tripartite synapse. Astrocytes account for over $90 \%$ of the uptake and metabolism of the glutamate [23]. Hence, the basic consequence of the loss of astrocytes is a reduction in glutamate uptake and thus, an excessive accumulation of glutamate in the synaptic cleft. As mentioned above, the glutamatergic predominance in the excitatory-inhibitory balance is postulated to be involved in the pathogenesis of depression. An increase in glutamate level was observed in the brains and cerebrospinal fluid of depressed patients [20] and inhibition of glutamatergic function had antidepressant effects [59]. In addition to the dysregulation of synaptic function, loss of glial cells could also underlie subsequent atrophy of neurons caused by stress and depression. A reduced number of GABAergic interneurons in the prefrontal cortex (PFC) of depressed patients as well as in rat's chronic stress model has been found recently [5, 30, 58]. These interneurons provide tonic inhibitory control over the firing of excitatory glutamatergic neurons, therefore, their degeneration may enhance glutamate/GABA unbalance induced by glial loss. Indeed, functional disruption of GABA transmission and decreased GABA levels were observed in depressed patients by magnetic resonance spectroscopy studies [48]. 
The role of astrocytic cell death or dysfunction in pathogenesis of depression has been confirmed by experimental studies in animals. A decline in the number of astrocytes in the prefrontal cortex was found in rats subjected to chronic unpredictable stress - one of the generally accepted animal models of depression [3, 4]. Chronic unpredictable stress was also found to induce a decrease in density of GFAP immunoreactive astrocytes and in GFAP protein level in the rat hippocampus [29] and in the hippocampus and prefrontal cortex [28]. Moreover, both astrocytic pathology and depression-like behavior were reversed by antidepressants clomipramine [29] or fluoxetine and magnolol [28]. Also studies employing others animal models of depression: psychosocial stress [13] or maternal deprivation [27] support the hypothesis that astrocytic pathology is associated with mood disorders.

The cause of glial cell loss in depression and in animal models of depression is not clear. We can only hypothesize that it may be the effect of environmental influence (stress, toxins) or/and genetic predisposition. The possibility of genetic basis is indicated by the results of Gosselin et al. [18], who investigated the expression of astrocytic markers GFAP in various brain regions in Wistar-Kyoto (WKY) rat strain in comparison to Sprague-Dawley (SD) rats. It is worth mentioning that WKY rats are commonly used as a model for anxiety and depression, as they are especially susceptible to stress. Those authors found slightly, but significantly lower density of GFAP-ir cells in the prefrontal cortical regions, basolateral amygdala and hippocampus CA3 and dentate area in WKY rats in comparison to SD rats. No statistical difference was found in other brain regions analyzed (insular cortex, somatosensory cortex, CA1 and callosal white matter). On the other hand, S100B level was similar in both strains of rats but double immunofluorescence studies have shown a good co-localization of GFAP and S100B expression in SD rats, whereas some S100B expressing astrocytes were devoid of GFAP in WKY rats.

Astrocytes may be also regulated by neurotransmitters. The effects of monoaminergic transmission on astrocytes has been postulated, as $\beta$-adrenergic receptor activation increases GFAP immunoreactivity and serotonin decreases them (according to [33]). Prolonged stress and chronic corticosterone treatment were reported to decrease GFAP mRNA in the cortex and hippocampus [36]. The depression is also con- nected with decreases in neurotrophic factors such as brain derived neurotrophic factor (BDNF), fibroblast growth factor 2 (FGF2) or glial cell line-derived neurotrophic factor (GDNF) [44], but it is not clear if it is a cause or result of astrocytic pathology. Reduction in glial density paralleled by an increase in the size of glial nuclei suggests that some compensatory mechanisms might be taking place. It may be speculated that if the number of normally functioning glial cells decreased, the survivor astrocytes are forced to work harder to support the metabolic needs and in consequence their nuclei are enlarged and changed (according to [42]).

\section{Impairment of astrocytes as a model of depression}

The idea that glial degeneration or dysfunction, especially evidenced in astrocytes, is very important for the development of depressive behavior constituted a starting point for developing new models of depression based on the dysregulation of Glu/GABA balance by impairment of the function of astrocytes. As the uptake of released glutamate by astrocytes in glutamatergic tripartite synapse has a fundamental role in this balance, some studies were focused on specific glial glutamate transporters. Postmortem studies of depressed subjects showed downregulation of genes coding for two excitatory amino acids transporters predominantly expressed in astrocytes in the cingulate and prefrontal cortex [12]. The reduced glutamate transporter expression and activity, especially GLT1 a specific glutamate transporter in rodents, was reported to occur in the hippocampus in the mouse stress model of depression induced by corticosterone exposure and additionally in the occipital and retrosplenial cortex in learned helpless rats, an animal model of depression [60]. A blockade of astrocytic glutamate uptake in the amygdala, a limbic structure engaged in mood regulation and anxiety, has been reported to decrease social exploration and disrupt circadian rhythms, the behavioral effects characteristic of depressive-like symptoms [26]. Central, intracerebroventricular injection of dihydrokainate (DHK), a specific inhibitor of astrocytic glutamate transporter (GLT-1) induced in rats signs of anhedonia observed in the intracranial self-stimulation test and cognitive 
impairment [6]. Similar effects were observed after DHK injection into the prefrontal cortex but the anhedonia in that experiments was measured also in the sucrose consumption test [23].

Another set of experiments examined astrocyte ablation in the prefrontal cortex (PFC) as a model of depression. Already in 1971 and in later years, it was shown that $\alpha$-aminoadipic acid (AAA), a six carbon homologue of glutamate, is a specific gliotoxic agent both in vivo and in vitro [21, 38, 39]. The AAA occurs naturally in the brain as an intermediate product of lysine metabolism and differs from glutamate by a single methylene group. When given as an exogenous molecule, it is taken up specifically by astrocytes and accumulating in these cells exerts its gliotoxic effect by interference with protein synthesis $[8,37]$. The exact mechanism underlying the AAA-induced gliotoxicity and its specificity is not known but it is supposed to be connected with the presence of an extremely potent $\mathrm{Na}^{+}$dependent carrier system for glutamate in the astrocytic membrane. This system has been shown to transport not only L-glutamate but also other related amino acids, like D- and L-aspartate and might also transport AAA as a close structural analog of glutamate [22]. The role of the $\mathrm{Na}^{+}$dependent glutamate uptake system into glial cells for transporting AAA into astrocytes was also confirmed by electrophysiological studies in vitro [40]. Studies on the effects of D- and L-isomers or racemic form of AAA gave conflicting, heterogeneous results. When administered subcutaneously into infant mice, it exerted a toxic effects in the arcuate hypothalamic nucleus, whereas D-AAA and D,L-AAA induced, respectively, a mild to extreme gliotoxic but not neurotoxic damage and L-AAA induced not only gliotoxic but also some neurotoxic changes, moreover, D-isomer had even antineurotoxic properties [38]. Similar results were obtained in astrocytes or mixed neuronal/astrocyte cultures [22] but additionally D-isomer appeared to be toxic only for mitotic cells [8]. Summing up all those results, it can be assumed that D,L-and L-AAA may be a good specific tool for astrocyte elimination in the brain tissue. The effect of AAA on astrocytes was also studied in adult rats after intracerebral injection into different brain structures. Single L-AAA injection into the amygdala induced loss of astrocytes and their markers (GFAP, S-100) in the wide area around the injection site, while neurons remained undamaged [24]. Astroglial degenerations were also observed after L-AAA injection into the striatum [52], substantia nigra and locus coeruleus [11]. On the other hand, Saffran and Crutcher [46] failed to observe gliotoxic properties of L-AAA or D,L-AAA in the hippocampus and striatum after injections into those structures.

A possibility of using astrocyte ablation as an experimental model of depression was presented for the first time by Banasr and Duman [4]. They demonstrated that a double microinjection of the specific astrocytic toxin, L-AAA, into the rat medial prefrontal cortex was sufficient to induce depressive-like behavior similar to that observed after chronic unpredictable stress, a generally accepted model of depression. A decrease in sucrose preference and increase in immobility time in the forced swim test were found after the toxin administration/infusion and moreover, a number of astrocytes stained with GFAP was significantly diminished in PFC. No depressive-like behavioral changes were observed after neurotoxin ibotenate infusion into the same region of PFC, which indicates that the effects are specifically connected with glial ablation. The specificity of astrocyte loss in the PFC after L-AAA toxin was confirmed also by immunohistochemical staining in which NeuN-immunoreactive neurons did not disappear after the toxin [4].

In our preliminary study, we have investigated whether this model may be useful for study of the antidepressant effects (paper in preparation). The doses and time schedule of our experiments were based on the paper of Banasr and Duman [4] but we used not only L-AAA but also the racemic form. The astrocytic toxin L-or D,L-AAA, was microinjected twice, bilaterally into the rat medial prefrontal cortex (PFC). Depressive-like behavior was assessed by a forced swim test (FST) on day 5 of the experiment. To verify whether this glial ablation model may be a useful animal model of depression, some rats were additionally treated with the classical antidepressant - imipramine. In short, imipramine reversed the behavioral effects of both toxins, which seems to confirm that it may be a good experimental model of depression.

We also checked astrocyte depletion in the PFC after the toxins. Histological verifications of the injection sites and immunohistochemical staining for the astrocytic marker, GFAP, were carried out. The stereological counting of the GFAP positive cells in the medial PFC confirmed the degeneration of astrocytes in this structure. Also western blot analysis performed at the same time after toxin injection, showed a significant decrease in the GFAP level in the PFC of toxin-treated rats but not in the caudal cortical areas, 
hippocampus and amygdala. A very interesting finding in our study is that the antidepressant imipramine not only reversed behavioral effects of toxins but also prevented (or partially inhibited) astrocyte degenerations, which was evidenced both by stereological counting and by western blot analysis. This parallelism of action of antidepressant both on normalizing the behavior and expression of astrocytes strengthens the argument for an important role of the loss of astrocytes in the prefrontal cortex in developing of depressive-like behavioral dysfunction.

\section{Conclusion}

The obtained results suggest an important role of astrocytes in the prefrontal cortex in mood regulation. Moreover, they indicate that the degeneration of astrocytes in this structure may be a useful animal model of depression especially for studying antidepresssant potential of compounds counteracting glutamatergic overexcitation. Indeed, our preliminary results indicate that compounds inhibiting glutamatergic transmission either via metabotropic glutamate receptors or via peptidergic mechanisms, which are postulated to be effective as antidepressant or anxiolytic agents, may prevent depressive-like behavior induced by AAA. However, further studies are needed to evaluate a potential therapeutic role of the antidepressant compounds on the basis of results obtained in the astrocyte ablation model.

\section{Acknowledgment:}

This study was supported by grant

„Depression-mechanisms-therapy” POIG.01.01.02-12-004/09.

\section{References:}

1. Altshuler LL, Abulseoud OA, Foland-Ross L, Bartzokis G, Chang S, Mintz J, Hellemann G, Vinters HV: Amygdala astrocyte reduction in subjects with major depressive disorder but not bipolar disorder. Bipolar Disord, 2010, 12, 541-549.

2. Araque A, Parpura V, Sanzgiri RP, Haydon PG: Tripartite synapses: glia, the unacknowledged partner. Trends Neurosci, 1999, 22, 208-215.
3. Banasr M, Chowdhury GM, Terwilliger R, Newton SS, Duman RS, Behar KL, Sanacora G: Glial pathology in an animal model of depression: reversal of stressinduced cellular, metabolic and behavioral deficits by the glutamate-modulating drug riluzole. Mol Psychiatry, 2010, 15, 501-511.

4. Banasr M, Duman RS: Glial loss in the prefrontal cortex is sufficient to induce depressive-like behaviors. Biol Psychiatry, 2008, 64, 863-870.

5. Banasr M, Dwyer JM, Duman RS: Cell atrophy and loss in depression: reversal by antidepressant treatment. Curr Opin Cell Biol, 2011, 23, 730-737.

6. Bechtholt-Gompf AJ, Walther HV, Adams MA, Carlezon WA Jr, Ongür D, Cohen BM: Blockade of astrocytic glutamate uptake in rats induces signs of anhedonia and impaired spatial memory. Neuropsychopharmacology, 2010, 35, 2049-2059.

7. Bowley MP, Drevets WC, Ongür D, Price JL: Low glial numbers in the amygdala in major depressive disorder. Biol Psychiatry, 2002, 52, 404-412.

8. Brown DR, Kretzschmar HA: The glio-toxic mechanism of a-aminoadipic acid on cultured astrocytes. J Neurocytol, 1998, 27, 109-118. Erratum in: J Neurocytol, 1998, 27, 281, 294.

9. Bunney BG, Bunney WE: Rapid-acting antidepressant strategies: mechanisms of action. Int J Neuropsychopharmacol, 2012, 15, 695-713.

10. Catena-Dell'osso M, Fagiolini A, Rotella F, Baroni S, Marazziti D: Glutamate system as target for development of novel antidepressants. CNS Spectr, 2013, 18, 188-198.

11. Chang FW, Wang SD, Lu KT, Lee EH: Differential interactive effects of gliotoxin and MPTP in the substantia nigra and the locus coeruleus in BALB/c mice. Brain Res Bull, 1993, 31, 253-266.

12. Chaudhry FA, Lehre KP, van Lookeren Campagne M, Ottersen OP, Danbolt NC, Storm-Mathisen J: Glutamate transporters in glial plasma membranes: highly differentiated localizations revealed by quantitative ultrastructural immunocytochemistry. Neuron, 1995, 15, 711-720.

13. Czéh B, Simon M, Schmelting B, Hiemke C, Fuchs E: Astroglial plasticity in the hippocampus is affected by chronic psychosocial stress and concomitant fluoxetine treatment. Neuropsychopharmacology, 2006, 31, $1616-1626$.

14. Damadzic R, Bigelow LB, Krimer LS, Goldenson DA, Saunders RC, Kleinman JE, Herman MM. A quantitative immunohistochemical study of astrocytes in the entorhinal cortex in schizophrenia, bipolar disorder and major depression: absence of significant astrocytosis. Brain Res Bull, 2001, 55, 611-618.

15. Edgar N, Sibille E: A putative functional role for oligodendrocytes in mood regulation. Transl Psychiatry, 2012, 2, e109.

16. Fatemi SH, Laurence JA, Araghi-Niknam M, Stary JM, Schulz SC, Lee S, Gottesman II: Glial fibrillary acidic protein is reduced in cerebellum of subjects with major depression, but not schizophrenia. Schizophr Res, 2004, 69, 317-323.

17. Feyissa AM, Chandran A, Stockmeier CA, Karolewicz B: Reduced levels of NR2A and NR2B subunits of NMDA receptor and PSD-95 in the prefrontal cortex in 
major depression. Prog Neuropsychopharmacol Biol Psychiatry, 2009, 33, 70-75.

18. Gosselin RD, Gibney S, O’Malley D, Dinan TG, Cryan JF: Region specific decrease in glial fibrillary acidic protein immunoreactivity in the brain of a rat model of depression. Neuroscience, 2009, 159, 915-925.

19. Hamidi M, Drevets WC, Price JL: Glial reduction in amygdala in major depressive disorder is due to oligodendrocytes. Biol Psychiatry, 2004, 55, 563-569.

20. Hashimoto K, Sawa A, Iyo M: Increased levels of glutamate in brains from patients with mood disorders. Biol Psychiatry, 2007, 62, 1310-1316.

21. Huck S, Grass F, Hatten ME: Gliotoxic effects of $\alpha$-aminoadipic acid on monolayer cultures of dissociated postnatal mouse cerebellum. Neuroscience, 1984, 12, 783-791.

22. Huck S, Grass F, Hörtnagl H: The glutamate analogue a-aminoadipic acid is taken up by astrocytes before exerting its gliotoxic effect in vitro. J Neurosci, 1984, 4, 2650-2657.

23. John CS, Smith KL, Van't Veer A, Gompf HS, Carlezon WA Jr, Cohen BM, Öngür D, Bechtholt-Gompf AJ: Blockade of astrocytic glutamate uptake in the prefrontal cortex induces anhedonia. Neuropsychopharmacology, 2012, 37, 2467-2475.

24. Khurgel M, Koo AC, Ivy GO: Selective ablation of astrocytes by intracerebral injections of $\alpha$-aminoadipate. Glia, 1996, 16, 351-358.

25. Largo C, Cuevas P, Somjen GG, Martín del Río R, Herreras $\mathrm{O}$ : The effect of depressing glial function in rat brain in situ on ion homeostasis, synaptic transmission, and neuron survival. J Neurosci, 1996, 16, 1219-1229.

26. Lee Y, Gaskins D, Anand A, Shekhar A: Glia mechanisms in mood regulation: a novel model of mood disorders. Psychopharmacology (Berl), 2007, 191, 55-65.

27. Leventopoulos M, Rüedi-Bettschen D, Knuesel I, Feldon J, Pryce CR, Opacka-Juffry J: Long-term effects of early life deprivation on brain glia in Fischer rats. Brain Res, 2007, 1142, 119-126.

28. Li LF, Yang J, Ma SP, Qu R: Magnolol treatment reversed the glial pathology in an unpredictable chronic mild stress-induced rat model of depression. Eur J Pharmacol, 2013, 711, 42-49.

29. Liu Q, Li B, Zhu HY, Wang YQ, Yu J, Wu GC. Clomipramine treatment reversed the glial pathology in a chronic unpredictable stress-induced rat model of depression. Eur Neuropsychopharmacol, 2009, 19, 796-805.

30. Maciag D, Hughes J, O'Dwyer G, Pride Y, Stockmeier CA, Sanacora G, Rajkowska G: Reduced density of calbindin immunoreactive GABAergic neurons in the occipital cortex in major depression: relevance to neuroimaging studies. Biol Psychiatry, 2010, 67, 465-470.

31. Manji HK, Drevets WC, Charney DS: The cellular neurobiology of depression. Nat Med, 2001, 7, 541-547.

32. Massart R, Mongeau R, Lanfumey L: Beyond the monoaminergic hypothesis: neuroplasticity and epigenetic changes in a transgenic mouse model of depression. Philos Trans R Soc Lond B Biol Sci, 2012, 367, 2485-2494.

33. Miguel-Hidalgo JJ, Baucom C, Dilley G, Overholser JC, Meltzer HY, Stockmeier CA, Rajkowska G: Glial fibrillary acidic protein immunoreactivity in the prefrontal cortex distinguishes younger from older adults in major depressive disorder. Biol Psychiatry, 2000, 48, 861-873.

34. Miguel-Hidalgo JJ, Rajkowska G: Comparison of prefrontal cell pathology between depression and alcohol dependence. J Psychiatr Res, 2003, 37, 411-420.

35. Nestler EJ, Gould E, Manji H, Buncan M, Duman RS, Greshenfeld HK, Hen R et al.: Preclinical models: status of basic research in depression. Biol Psychiatry, 2002, 52, 503-528.

36. Nichols NR, Osterburg HH, Masters JN, Millar SL, Finch CE: Messenger RNA for glial fibrillary acidic protein is decreased in rat brain following acute and chronic corticosterone treatment. Brain Res Mol Brain Res, 1990, 7, 1-7.

37. Nishimura RN, Santos D, Fu ST, Dwyer BE: Induction of cell death by L-alpha-aminoadipic acid exposure in cultured rat astrocytes: relationship to protein synthesis. Neurotoxicology, 2000, 21, 313-320.

38. Olney JW, de Gubareff T, Collins JF: Stereospecificity of the gliotoxic and anti-neurotoxic actions of $\alpha$-aminoadipate. Neurosci Lett, 1980, 19, 277-282.

39. Olney JW, Ho OL, Rhee V: Cytotoxic effects of acidic and sulphur containing amino acids on the infant mouse central nervous system. Exp Brain Res, 1971, 14, 61-76.

40. Pannicke T, Stabel J, Heinemann U, Reichelt W: $\alpha$-Aminoadipic acid blocks the $\mathrm{Na}^{+}$-dependent glutamate transport into acutely isolated Müller glial cells from guinea pig retina. Pflugers Arch, 1994, 429, 140-142.

41. Paul IA, Skolnick P: Glutamate and depression: clinical and preclinical studies. Ann NY Acad Sci, 2003, 1003, 250-272.

42. Rajkowska G, Miguel-Hidalgo JJ: Gliogenesis and glial pathology in depression. CNS Neurol Disord Drug Targets, 2007, 6, 219-233.

43. Rajkowska G, Miguel-Hidalgo JJ, Wei J, Dilley G, Pittman SD, Meltzer HY, Overholser JC et al.: Morphometric evidence for neuronal and glial prefrontal cell pathology in major depression. Biol Psychiatry, 1999, 45, 1085-1098.

44. Rajkowska G, Stockmeier CA: Astrocyte pathology in major depressive disorder: insights from human postmortem brain tissue. Curr Drug Targets, 2013, 14, $1225-1236$.

45. Rodnight RB, Gottfried C: Morphological plasticity of rodent astroglia. J Neurochem, 2013, 124, 263-275.

46. Saffran BN, Crutcher KA: Putative gliotoxin, $\alpha$-aminoadipic acid, fails to kill hippocampal astrocytes in vivo. Neurosci Lett, 1987, 81, 215-220.

47. Sanacora G, Banasr M: From pathophysiology to novel antidepressant drugs: glial contributions to the pathology and treatment of mood disorders. Biol Psychiatry, 2013, $73,1172-1179$.

48. Sanacora G, Saricicek A: GABAergic contributions to the pathophysiology of depression and the mechanism of antidepressant action. CNS Neurol Disord Drug Targets, 2007, 6, 127-140.

49. Schroeter ML, Abdul-Khaliq H, Diefenbacher A, Blasig IE: S100B is increased in mood disorders and may be reduced by antidepressive treatment. Neuroreport, 2002, 13, 1675-1678.

50. Si X, Miguel-Hidalgo JJ, O’Dwyer G, Stockmeier CA, Rajkowska G: Age-dependent reductions in the level of 
glial fibrillary acidic protein in the prefrontal cortex in major depression. Neuropsychopharmacology, 2004, 29 , 2088-2096.

51. Skolnick P, Layer RT, Popik P, Nowak G, Paul IA, Trullas R: Adaptation of N-methyl-D-aspartate (NMDA) receptors following antidepressant treatment: implications for the pharmacotherapy of depression. Pharmacopsychiatry, 1996, 29, 23-26.

52. Takada M, Hattori T: Fine structural changes in the rat brain after local injections of gliotoxin, $\alpha$-aminoadipic acid. Histol Histopathol, 1986, 1, 271-275.

53. Trullas R, Skolnick P: Functional antagonists at the NMDA receptor complex exhibit antidepressant actions. Eur J Pharmacol, 1990, 185, 1-10.

54. Uranova NA, Vostrikov VM, Orlovskaya DD, Rachmanova VI: Oligodendroglial density in the prefrontal cortex in schizophrenia and mood disorders: a study from the Stanley Neuropathology Consortium. Schizophr Res, 2004, 67, 269-275.

55. Vostrikov VM, Uranova NA, Orlovskaya DD: Deficit of perineuronal oligodendrocytes in the prefrontal cortex in schizophrenia and mood disorders. Schizophr Res, 2007, 94, 273-280.

56. Wierońska JM, Pilc A: Metabotropic glutamate receptors in the tripartite synapse as a target for new psychotropic drugs. Neurochem Int, 2009, 55, 85-97.
57. Yang J, Shen J: In vivo evidence for reduced cortical glutamate-glutamine cycling in rats treated with the antidepressant/antipanic drug phenelzine. Neuroscience, 2005, 135, 927-937.

58. Zadrożna M, Nowak B, Łasoń-Tyburkiewicz M, Wolak M, Sowa-Kućma M, Papp M, Ossowska G et al.: Different pattern of changes in calcium binding proteins immunoreactivity in the medial prefrontal cortex of rats exposed to stress models of depression. Pharmacol Rep, 2011, 63, 1539-1546.

59. Zarate CA Jr, Singh JB, Carlson PJ, Brutsche NE, Ameli R, Luckenbaugh DA, Charney DS, Manji HK: A randomized trial of an N-methyl-D-aspartate antagonist in treatment-resistant major depression. Arch Gen Psychiatry, 2006, 63, 856-864.

60. Zink M, Vollmayr B, Gebicke-Haerter PJ, Henn FA: Reduced expression of glutamate transporters vGluT1, EAAT2 and EAAT4 in learned helpless rats, an animal model of depression. Neuropharmacology, 2010, 58, $465-473$.

Received: July 30, 2013; in the revised form: September 20, 2013 ; accepted: September 24, 2013. 